\title{
SIMULTANEOUS OPERATION OF A LIQUID ARGON DETECTOR AS BUBBLE CHAMBER AND CALORIMETER
}

\author{
J.C. BERSET, M. BURNS, G. HARIGEL, J. LINDSAY, G. LINSER and F. SCHENK \\ CERN, Geneta, Switzertand
}

Received 27 April 1982

The first successful operation of a new detector, filled with liquid argon is described. It can be used simultaneously as bubsle chamber and calorimeter, and may find interesting applications at high-energy accelerators.

\section{Introduction}

The new generation of fixed-target accelerators in the $\mathrm{TeV}$ energy range requires multiton detectors. which give specific information on the topology of events. allow particle identification. momentum determination and provide timing information. This is of special interest for neutrino interactions, containing strange particles and multileptons. where one or more of the leptons are electrons [1]. For financial, technical and safety reasons cryogenic bubble chambers filled with hydrogen. deuterium or neon-hydrogen mixtures can probably not be built with much larger volumes than some 35 $\mathrm{m}^{3}$ (Big European Bubble Chamber at CERN. 15-fo Bubble Chamber at Fermilab). Another difficulty lies in their limited precision in measuring the momentum of a very energetic electron [2] (fig. 1) or of a hadronic shower. An attractive way out of these problems could be the use of liquid argon in a new type of detector. which can be operated simultaneously in the bubble chamber and calorimeter modes. The relatively high specific density and short interaction length of argon makes muon identification inside large volumes fairly easy. Furthermore, timing information on (rare) interactions can be reliably and rapidly obtained from the scintillation pulse produced during the passage of particles [3]. This signal can be used to trigger the flash of the bubble chamber, provide a gate for the charge collection, and give even crude results on energy contained in heavy showers and thus complement calorimetry from the charge sensitive amplifier.

Such a detector may also find application at colliding beam machines, e.g. at the SLAC linear collider, where it could be built around the vacuum tube of the interaction region, or it could be used sven in proton decay experiments, provided that its volume can be made large enough.

The feasibility of producing good particle tracks in a

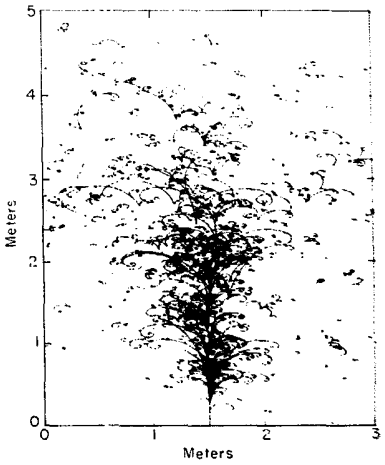

Fig. 1. Monte Carlo simulated electron shower (primary ensigy $5 f)$ (ieV) in a heavy neon/hydrogen mixture $\left(x_{0}=00 \mathrm{cmad}\right.$ magnetic field $30 \mathrm{kG}[2]$.

2.7 f argon test chamber was demonstrated receatly [4] In the present paper we show that chatge collectioa with reasonable accuracy is possible diring bubble chamber expansions. The experimental set-up is dt scribed. results of measurements in a $200 \mathrm{MeV} / \mathrm{e}$ pioa test beam are given, and future improvemints in charge collection, mainly by employing the sciatillation pulse as a trigger, are discussed.

0167-5087/82/0000-0000/\$02.75 1982 North-Holand 

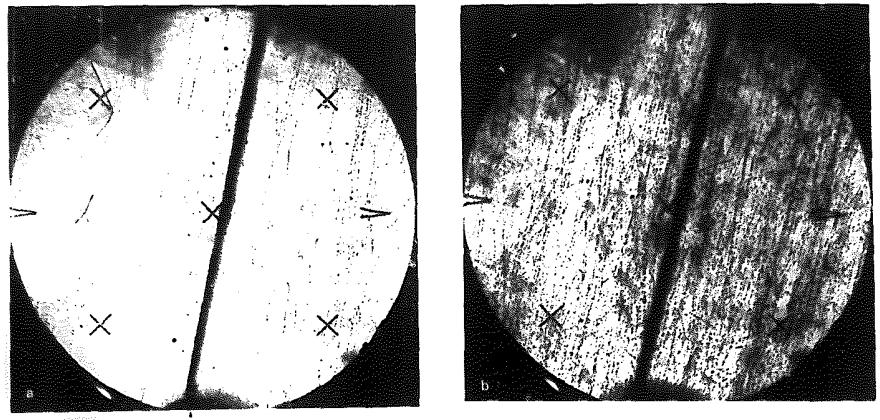

Fig. 2. Photos of $200 \mathrm{MeV} / \mathrm{c}$ pions in liquid argen at $135 \mathrm{~K}$. Bubble density $(18 . \div 2$ ) bubbles $/ \mathrm{cm}$. bubble size $\varnothing \equiv 0.3 \mathrm{~mm}$. Charge collection wire with $\varnothing<1 \mathrm{~mm}$ in the centre of the bubble chamber: (a) low heam intensity $(-20$ tracks). (b) mediun beam intensity $(\sim 60$ tracks). (c) figh heam intensity $(-150$ iracks).

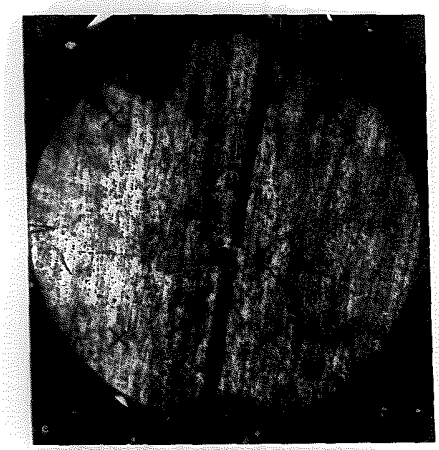

time interval between successive pulses is $\sim 5 \mathrm{~ms}$, well matched to the sensitive time of approximately $4 \mathrm{~ms}$ during a $15 \mathrm{~ms}$ expansion-recompression cycle of the bubble chamber: only one particle burst can produce tracks during such a cycle. During short running periods the accelerator was operated with only one particle vurst every $2 \mathrm{~s}$, which is the present repetition rate of our bubble chamber. The duration of the particle burst is $\sim 40 \mu \mathrm{s}$ fwhm, having a Gaussian-like intensity distribution, a microstructure of $60 \mathrm{~ns}$, and a jitter of $(10 \mathrm{~ns}$.

The beam intensity is monitored by coincidence counters in front of the vacuum tank of the detector. The diameter of the beam is chosen to be $\$ 8 \mathrm{~cm}$, so that the particles fill homogeneously the visible volume of the chamber. Those particles, going throug:n the centre of the cylindrical chamber (axis perpendicular to the beam) have to penetrate a $2 \mathrm{~mm}$ aluminium vac sum tank window and $11 \mathrm{~mm}$ of stainless steel of the chamber vessel. This causes a momentum loss of $\sim 10 \%$ and a mean scattering angle of $\sim 0.5^{\circ}$ puior to the entrance. into the liquid. These values are larger by a factor of up to 1.5 in the border region of the visible volume. Th: occasional use of collimators allowed a reduction of the beam profile to $2 \mathrm{~cm}$ and an adjustment of the beam position relative to the charge collection wire. 


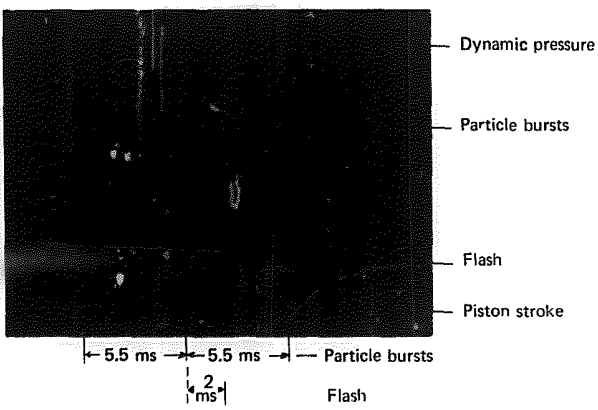

Fig. 3. Piston stroke (0.5 mm/division) and dynanic pressure ( -4 har/division): time stale: 2 ms divisim.

\subsection{The detector}

\subsection{The bubble chember}

The bubble chamber is described in detail in ref. 4 . It was operated with argon at a liquid temperature $T=$ ? $135 \mathrm{~K}$ (vapour ressure $P_{\mathrm{v}}=25.6 \mathrm{har}$ ) and static pressure $P,=26.5$ bar. With a volume change of $\Delta V / V \leqslant 1 \%$ ( $2 \mathrm{~mm}$ piston stroke) we obtained an expanded pressurc $P_{e}=10$ har (the piezoelectric pressure transducer lat not yet been precisely calibrated). The duration of the expansion-recompression cyck was $t_{\mathrm{c}}=15 \mathrm{~ms}$ unless otherwise stated. The beam was injected at the pressure minimum and the flash delay was $\Delta_{1}=1.5 \mathrm{~ms}$. Tracks were photographed with a single camera. The above conditions result in tracks with trubble sizes of $\leqq 0.3$ $\mathrm{mm}$ and a bubble density of $(18+2)$ bubbles $/ \mathrm{cm}$. the latter not being corrected for the unknown dip angles. The picture quality allowed a fairly reliable count of the number of particles going through the chamber up (w) - 100 tracks per photo. Typical photos are shown in figs. 2, the piston stroke and the dynamic pressure vs. time in fig. 3 .

Argon with a purity of $>99.9999 \%$, as guaranted by the manufacturer (contamination with $\mathrm{O}_{2}<0.1, \mathrm{~N}_{2}<$ 0.3. $\mathrm{H}_{2}<0.001, \mathrm{CH}_{4}<0.005, \mathrm{H}_{2} \mathrm{O}<1$ ppm). was used: no additional analysis of the gas was carried out.

2.2.2. The charge collection device inside the hubble shamber

Most of the physics aspects of liquid ionization detectors are described in great detail in the literature (e.g. refs. 5, 6, where extensive bibliographics are given), and recent results on drifting of electrom oner lirge distanses atre reported (e.g. refs. 7. 8). Therefore we describe here only our technictl lavout.

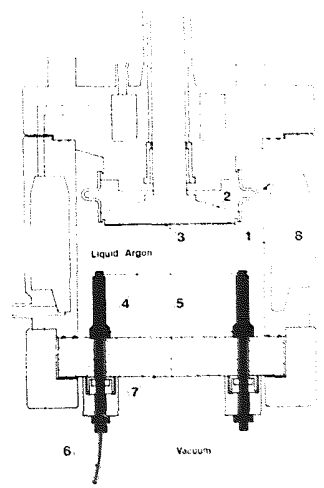

Fig. A. Verticial erews secton of the butble chamber wath shats

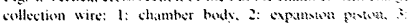

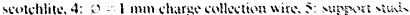

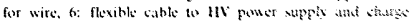

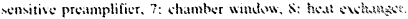


The positive high de voltage is brought from the outside through the vacuum tank of the detector to the wire inside the bubble chamber liquid. For reasons of mechanical simplicity and stability and in order to minimize optical and thermodynamic complications we have chosen the preliminary layout depicted in fig. 4 . Two holes, $10 \mathrm{~cm}$ apart. in the $70 \mathrm{~mm}$ thick bubble chamber window (BK7 glass), carry vacuum and pressure tight electric feedthroughs. which also serve as a ngid support for the $1 \mathrm{~mm}$ diameter charge collection wire. The wire is oriented parallel to the particle beam. One of the feedthroughs is connected to the high-voltage stabilized power supply (Fluke, $10 \mathrm{kV}$ maximum) and to the charge amplifier. which is also installed outside the vacuum tank to allow modifications during the tests. All connectors, especially inside the vacuum tank. are carefully shaped and polished to avoid corona discharges, which would produce undesirable electronic noise.

This simple arrangement inside the chamber volume, where charges are not only collected on the wire but also on its support structure, has the drawback that the electric field distribution is hard to calculate. This makes the calibration of the collected charges as function of the number of particles difficult; consequences will be discussed below. To circumvent some of these problems we plan to change this layout during forthcoming tests: but presumably at the expense of optical quality and increased parasitic boiling.

\subsubsection{The electronics and readout system}

No special electronics was constructed for this series of tests. The preamplifier, a charge sensitive FET input amplifier, had been previously used in trials of the Nal calorimeter for the axial field spectrometer at the CERN ISR [9]. A circuit is shown in fig. 5.

Fig. 6 shows the set-up for the charge collection during the beam spill of the accelerator. A trigger from the synchrocyclotron was suitably delayed so that a scaler could be gated open during the particle burst. Two coincidence counters in the beam were used to select true beam particles passing through the argon bubble chamber. Since the beam spill time is approximately $40 \mu \mathrm{s}$ fwhm and it has a Gaussian-like intensity

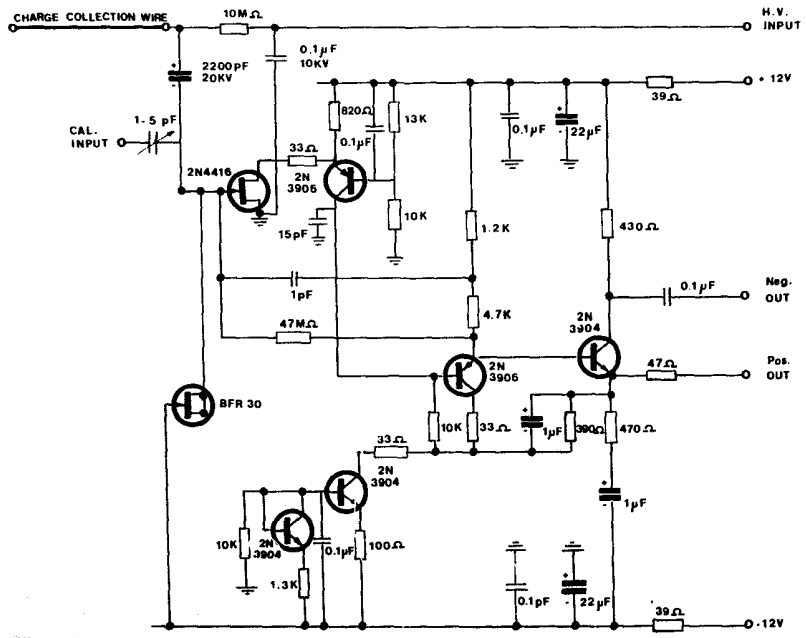

Fig. S. Miniaturized charge sensitive preamplifier. 

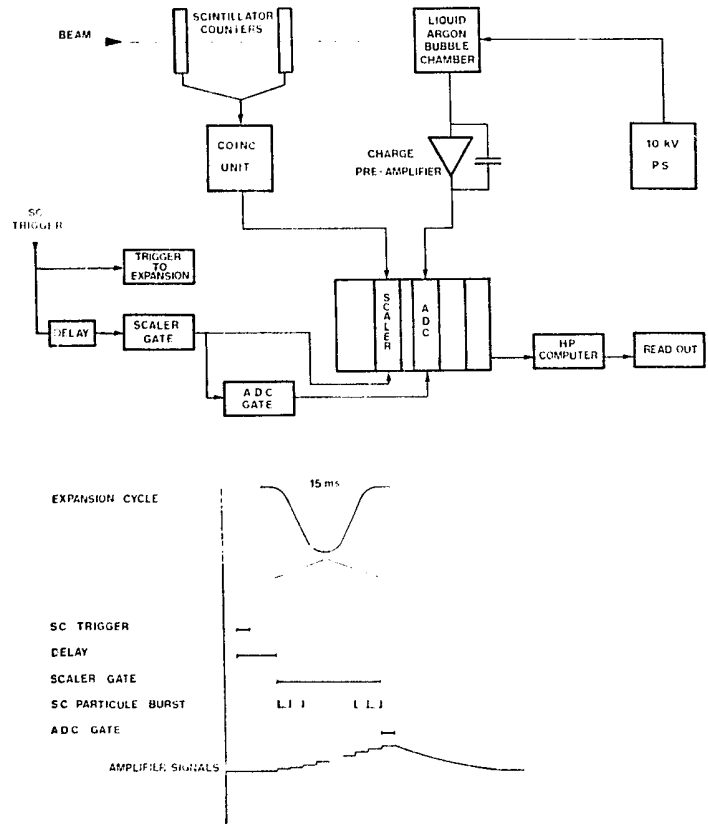

Fig. 6. Electronic configuration for charge collection, including interfate to computer.

distribution, there is a charge build-up in the charge sensitive preamplifier because of its long time constant $(\sim 50 \mu \mathrm{s})$. Fig. 6 shows an idealized wave form pattern. At the end of the scaler gate the preamplifier ouiput voltage was sampled by a charge-sensitive ADC. Scaler and $A D C$ values were read out via CAMAC to a Hewlett-Packard computer.

A second set-up did not require the accelerator bei'،n and was used to show that the signal due to the variation of the chamber capacitance during an expansion cycle could be filtered out so that it did not contribute io the charge created by beam particles. A precision pulse generator was used to inject the charge into the preamplifier via the calibration input. The output signal was shaped by a double differentiating amplifier with a $600 \mathrm{~ns}$ shaping time constant. This shaping amplifer is a bipolar RC clipped circuit, which had been previoualy used with a liquid argon calorimeter [10]. The height of thi: signal was measured by an $A D C$ and read cat vis CAMAC.

\section{Experimental results}

\section{3.l. Noise sources}

The primary aim of our tests was to show that the mechanical expansion of the bubble chamber is compatible with the collection of free charges producel durias 


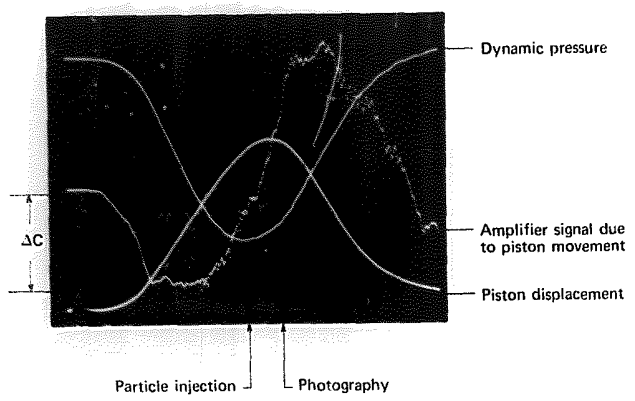

Fig. 7. Signal from charge sensitive preamplifier ( $1 \mathrm{~V} /$ division), $10 \mathrm{kV}$ on wire. produced by change of capacitanee during bubble chamber expansion. escle duration $15 \mathrm{~ms}$, together with piston displacement $(0.5 \mathrm{~mm}$ division). and dynamic pressure $(\sim 4$ har /division). Time scale: $2 \mathrm{~ms} /$ diviston, $\Delta C$ is the maximal amplitude of the unwanted signal prior to particle injection.

the passage of high energy particles. The main concern had to be, therefore, to identify unwanted electric, electronic and microphonic noise sources, which could coupie to the charge sensitive amplifier. Such disturbances, coming from the electric part of the hydraulic expansion system and from various power supplies were found and eliminated. The box. containing the charge amplifier and capacitors. is mechanically attached to the vacuum tank and screened against high-frequency noise. The vibration of the box. which is very small during the expansion of the chamber, does not affect the charge measurement. The movement of the charge collection wire relative to the chamber vessel during the expansion is not known but, considering its rigid support structure, should be very small. When all electric and electronic equipment and the detector was running, with $10 \mathrm{kV}$ on the charge collection wire, but without the mechanical expansion of the chamber, the remaining noise with a main frequency of $-250 \mathrm{~Hz}$, was $15 \mathrm{mV}_{(p p)}$, i.e. less than $0.5 \%$ of the total range of the preamplifer.

To produce good bubble tracks in our chamber the piston must be rapidly moved by $\sim 2 \mathrm{~mm}$. Considering the chamber height of $100 \mathrm{~mm}$, this displacement corresponds to a capacitance change of less than $2 \%$. The resulting unwanted signal on the charge sensitive amplifier is shown in fig. 7 for a $15 \mathrm{~ms}$ expansion-recompression cycle and a high voltage of $10 \mathrm{kV}$. This signal looks essentially like a derivative of the displacement curve. The amplitudes of the parasitic signai increase linearly with the applied HV, and their dependence upon the cycle duration is shown in fig. 8. The quite significant drop of this disturbance with increasing cycle duration favours the prospects for very large bubble chambers. There, longer duration of the cycle is required for various reasons, in particular for growing bubbles to the necessary larger sizes compared with those in our small test device, and because of pressure

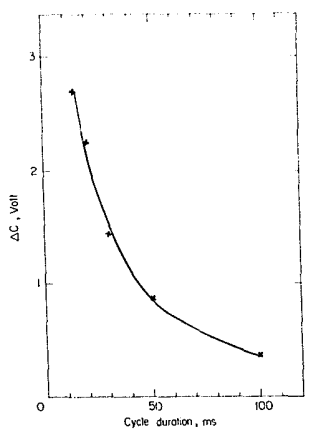

Fig. 8. $\Delta C$ (compare fig. 7) as function of the duration of the expansion cycle. 
homogeneity (sound velocity). At constant volume change. but increasing cycle duration, there is a decrease of the pressure drop due to the increased influence of parasitic boiling. In our chamber, this amounts to a $15 \%$ loss when the cycle length changes from 15 to $100 \mathrm{~ms}$. In spite of this fact, we still obtained sensitivity at $300 \mathrm{~ms}$ cycle duration. In muc , bigger chambers this drop should $b_{c}$ far less pronounced because of the more favourable vo'ume/surface ratio.

The infiuence of the change in capacitance appears to be less dramatic when we consider the following facts: (a) the piston is almost at rest when the pressure minimum is reached. (b) the disturbing signal is very reproducible for constant chamber conditions and (c) has its smallest : mplitude during the time of particle injection. and (d) the time interval between particle burst and collectior of all charges is relatively small and so is, the change ir the disturbing signal. However, to trigger a gate betw:en the charge amplifier and a double differentiating anplifier of suitable shaping time to collect all charges remains necessary for any precision measurement.

The unwanted signal from the change of capacitance between expansion bellows and wire, particular to our test set-up, can be largely suppressed in any future big ch.mber either by a non-conductive piston structure or/and by a screening grid between the moving part and the charge collector.

Another disturbance arises from the trigger pulse for the bubble chamber flash. This pulse is difficult to screen and it saturates the charge implifier $(\sim 7 \mathrm{~V})$; the signal decays within $0.5 \mathrm{~ms}$. However, this disturbance comes a long time (some milliseconds) after the gate for charge collection, and therefore cannot anymore influence the charge measurements.

\subsection{Performance of the amplifie, . . calbration pulse}

A precision pulse generator was used to inject charges into the preamplifier via the calibration input. The preamplifier signal was shaped by a double differentiating amplifier, which happened to have a $600 \mathrm{~ns}$ shaping time constant. This time is well adapted for electron drift distances in the millimetre range. but has to be increased for physics experirients. when drift distances over centimetres or even a metre are envisaged. Here. our purpose is only to demonstrate that the signal duc to the variation of chamber capacitance during an $\mathrm{ex}$ pansion cycle could be filtered out so that it did not contribute to the charge created by particles.

The maximum pulse height of the injected signal was $6.3 \mathrm{mV}$ at $2 \mathrm{pF}$, which corresponds to $0.0125 \mathrm{pC}$. A similar charge would be produced by a minimum ionizing particle with $\sim 2 \mathrm{~cm}$ track length, if we use as mean energy loss by ionization $\overline{\Delta E}=2 \mathrm{MeV} / 8 \mathrm{~cm}^{2}$ ?, an argon density $\rho=1.0 \mathrm{~g} / \mathrm{cm}^{3}$ at $135 \mathrm{~K}$, and an average energy for forming an ion pair $\bar{E}=25 \mathrm{eV}$, resulting in $4 \times 10^{4}$ electrons $/ \mathrm{cm}$.

The height of the output signal was measured by an $A D C$ and read out via CAMAC. A series of 50 meatsurements were made per point. Each series was treated to give the mean deviation from the average valve. Measurements were made as follows (all numerical values in ADC units):

(a) Only the amplifier in circuit. i.e. no connection to the liquid argon chamber, with expansions, cycte duration $15 \mathrm{~ms}$. no high voltage on wire:

(i) charge injection at $p_{\min } \quad 612=4$ :

(ii) charge injection at half $\Delta P$ after $P_{\operatorname{man}} \quad 700 \div 4$.

(b) Liquid argon chamber connected to amplifer circuit. no expansions, $10 \mathrm{kV}$ on wire:

(i) motor of the hydraulic pump off $317=16$. (ii) motor of the hydraulic pump on $\quad 316=14$.

(c) Liquid argon chamber connected to amplifier sircuit, with expansions. cycle duration $15 \mathrm{~ms}, 10 \mathrm{kV}$ on wire:

(i) charge injection at maximum piston stroke

$323=13:$

(ii) charge injection at $P_{\min }$

$322-15:$

(iii)charge injection at half stroke before $P_{\min }$

(iv)charge injection at half stroke after $t_{\text {tain }}$

$312=14:$ $301=15$

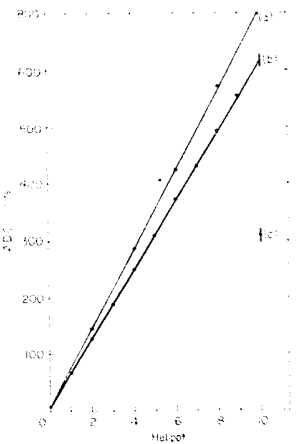

Fig. 9. Performance of the charge seasitive peabuplifise datade injection of ealibrated signals of vatious ampatuks chames:

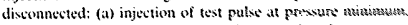

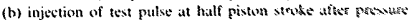
minimum; and thamber connected: (s) injotion of test putas at pressufe minimum. 
(d) liquid argen chamber connected to amplifier circatit. with expansions. crek dutation $80 \mathrm{~ms} .11 \mathrm{kV}$ an wire:

(i) sharge injection at maximum piston stroke

$311 \cdot 17$

The main features of this tests are: there is a linear relationship between injected and measured charges for conditions ati) and a(ii) (see also fig. 9). When the charge collection wire is elcetrically connected, its capacitance (22 pF meastared with liquid) reduces the output signal of the preamplifier as expected. The chamber cannot he exnsidered to be a gond Fariday citere. since the relative rms noise level increases from - $0.7 r_{r}$ to about $5 f_{i}$ after it is comolected with the charge sampling electronics. High frequency noise maty enter the liquid via the chamber window, cables for the pressure transducer, or via the high-tension wire. With expansions, the amptitucle of the signal is highest near the pressure minimum. and lower at hatf of the piston stroke on hoth sides of the minimum. This result maty be explained by the then more prominent clange of capactance produced by the piston movement (compare fig. 7). There was no significant change of the signal with different duration of the expansion eycle. probably because of the short shaping time constatlt.

Two preamplifters were tried which had gains differing bx a factor of 2.5: they buth showed similar features. The pedestal was - $20 \mathrm{ADC}$ units.

$\therefore$ Charge oblection from particles with a non-expanded stiumber

All approxinate 3:1 refation was established at fow heam intersities betwen the coincidence counters in from of the detector and the number of particle tracks phoiograpled with the bubble chamber camera. The highest number of tracks which could be reliably counted on film is -100 . However. the above relation is assumed to hold for much higher numbers.

The heam intensity is monitored by the coincidence counturs and was aiways stable over long time intervals within $6.5^{\circ}$; and $-3 \%$ for high and fow intensities. respectively: bowever. variations from pulse to pulse could attain some $\pm 30 \%$. A plot of the coincidence count: against the sealer readings shows linear behaviour.

The racks fill the visible volume of the bubble chamber almost homogeneously as deduced from the photos (compare figs. 2). The tracks are essentially parallel to the clarge collection wire.

Meatarements of the collected charges were made without expassion for four different beam intensities. during which the voltage was increased from 0 to +10 $\mathrm{hV}$ in steps of $1 \mathrm{kV}$. For the lower intensities 50 beam pulses were accunulated and registered with the computer, at the highest bean intensity only ten pulses. The

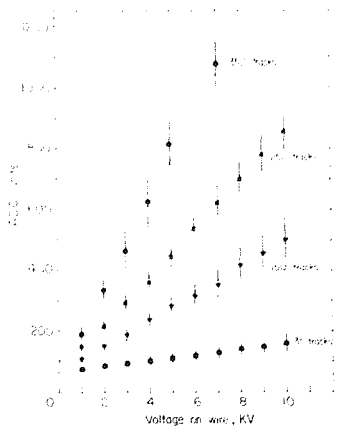

Fig. 10. ADC readings vs. high soltage. heam intensity as pirameter, withut expan:ion.
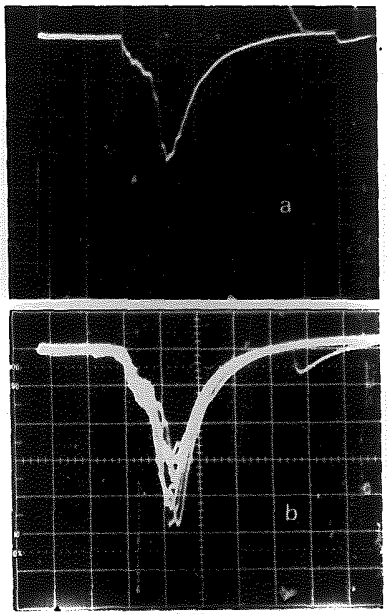

$\mathrm{Fg}$, 11. Typical signal from the charge sensitive preampliftes. -100 tsacks. without expansion: (a) 1 beam spill only (b) 10 heim spills superimposed; stales: $50 \mu \mathrm{s} /$ division. $50 \mathrm{mV} /$ division. 
results are depicted in fig. 10: amplitudes of the collected charges are given in $\mathrm{ADC}$ units with statistical errors only. The varsations at each measured point are essentialily due to the fluctuations in the beam intensity from pulse to pulse. and to variations of the maximum of the huild-up charge in the amplifier relative to the fixed position of the 100 ns gate to the charge sensitive ADC. Fig. 11(a) shows the charge build-up during one accelerator spill: fig. $1 /(b)$ when ten spills are superimposed on the oscilloscope. The relative uncertainties of $A D C$ readings increase from $8 \%$ at high beam intensities to $14 \%$ at low intensities. A linear fit is made through the measurements at each of the forur beam intensities. The noise level corresponds to $-50 \mathrm{ADC}$ units.

For different heam intensities the ratio of the : pes of collected chatge is in gend agreement with the corresponding ratio of the coincidence counts. $i . c$. we measure real charges from beam particles. There seems to he a lirear dependency of collected charges upon the applied voltage up to $10 \mathrm{kV}$. At very high fields we would expect at deviation from this linearity and an asymptosic hehasviesur.

At prevent our results cats be explained only qualitatively hecause charge collection depends in a complex manner upon various parameters: (a) the electric field distribution. (h) the distance of particles from the collection electrode. and (c) the purity of the liquid.

In a crude approximation our detector has a cylindrical field distribution. where we may define a bubhle chamber "radisis" with $R=5 \mathrm{~cm}$, having in its centre a wire with $r .0 .05 \mathrm{~cm}$. When we apply at vollage of 10 $k \mathrm{~V}$. then the radial field would be $f(x)=2.2 \mathrm{kV}$. where $x$ is the distance from the wire in cm.

The drift velocity of electrons in liquid argen mats be described in good approximation for field strengths between 0.3 and $30 \mathrm{kV} / \mathrm{cm}$ by $[1] .12]$

$r_{1}[\mathrm{~cm} / \mathrm{s}]=2.1 \times 10^{6} \mathrm{E}^{1}$ !.

An increase of the applied voltage catuse a decrease of hoth the drift time and electron attachment on clecironegative impurities. The attenuation of sollected electrons can he expressed by $[1,3]$

$q(x)=g(0) \mathrm{e} \cdot t$.

where $l .(\mathrm{cm})=0.15 \frac{t(\mathrm{kV} \mathrm{cm})}{p\left(\mathrm{ppm} \mathrm{O}_{2}\right)}$

and $p=$ electronegative impurities concentration (manls oxygen).

Oxygen impurties in our chamber filling shoukl wot exceed $0.1 \mathrm{ppos}$. since this was guaranted by the manufacturer, and proper eare was taken during the purging of the chamber 10 avoid any additional contamination. Then, with $10 \mathrm{kV}$ on the wire stottoms created $1 \mathrm{~cm}$ away from it have a mean free path of
$L=3 \mathrm{~cm}$, i.e. at least 70 \% of these clectrom atis he Jetected.

Drifting electrons induce an image currene on the collection wire. depending at which distance the ate created. given by

$f(x)=\frac{r_{j}(x)}{x \ln (R x)}$

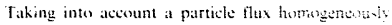
distributed in a plane perpendicular to the wire ant the above three relations, one may expect a linear incesase of the number of collecied electrons upon mereising voltage to $-10 \mathrm{kV}$. However. the position of the mathmum amplitude of the signal may shift someting in time, Any more quantiative statements requirs bo with a "pencil" heatn of very short spill furation. when can be injected into the chamber at various distansefrom the wire. Furthermore, an electrode arrangensus? giving a wetl-defined field distribution whla be at vatilgerous,

3.4. Charge collection from partales daring bothe shimher expunsions

Measurements of charge collection at the prestuts

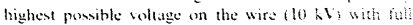
expansion of the shamber were matle. At some besm intensites seteral hundred photos were tation samest. tively.

Mont of the meavurements were mate at les and moderate beam intensities, mainly to study the senvitiv ily of enr device and its signal-to-notse ratio. The tewtio are plotted in $\mathrm{ADC}$ units versus be:m intensity fige 12 , For comparison we shom also the linesr dependence of

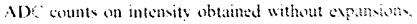
as extracted from fig. (i). Feg. I2 demunteats that

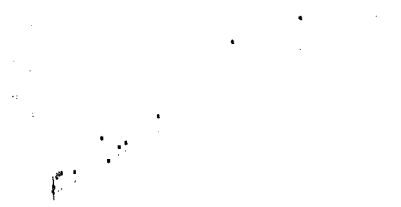

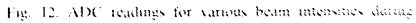

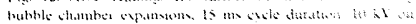

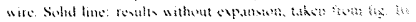


charge collection during expansion is possible, and compared to a quiet chamber, practically all ADC readings during expansion are higher, and their rms fluctuations are larger by a factor of two. The beam intensities at which photos $2(a-c)$ were taken are indicated.

No attempt is made at present to calibrate our measurements absolutely, mainly because of the long bean spill and of variations in beam intensity from spilt to spill.

In any future large chamber the time spread in the arrival of free electrons from a real event is expected to be smaller than our present beam spill: however, its value depends on the electrode spacing. The timing information from an event derived from its scintiliation signal should be used to initiate a gate between the charge sensitive preamplifier and a double differentiating amplifier with suitable shaping time, adjusted to collect all free electrons from such an event. The adverse influence of the change of capacitance during expansion can probably be considerably reduced also by a properly adapted cage. to be kept at ground potential, thus screening the piston movement; or by a piston made of non-conductive material. With these possible improvements in mind, we believe that the present disturbances can be substantially reduced.

\subsection{Electron drift and bubble formation process}

The presence of a high electric field $(+10 \mathrm{kV}$ on the wire and support stems, distance to the chamber body 5 and $1.5 \mathrm{~cm}$, respectively) appears to cause neither a noticeable reduction nor an increase of bubble densities along the particle track as compared to no-field conditions. However, with our single view optic, it is neither possible to determine the distance of tracks from the charge collection wire in the central part of the chamber, nor to see tracks between the support stems of the wire and the adjacent chamber wall, where the electric field is highest. As was pointed out by Hofmann and Hugentobler [14], it is not yet shown experimentally in a conclusive manner, whether the bubble formation by 8-efectrons is due to an accumulation of ions of the same charge and their repulsive forces, or due to local heat concintration produced by ion recombination. As suggested to us by Doke [15], this question might be clarified during a repetition of our experiment, to be done at fields higher than about $3 \mathrm{kV} / \mathrm{cm}$, where ion pairs can be separated, and by using at the same time a better defined field geometry. A.t present we can try only 10 explain the absence of obvious variations in bubble densities by discussing two possible effects of electric fields tson the bubble formation: (a) change of the direction and speed of 8 -electrons and (b) escape of electrons and holes from recombination.

(a) In order to grow bubbles along a particle track to visible size, the necessary condition is, that the initial bubble formed by $\delta$-electrons has to attain at least the critical size. The critical radius $r_{c}$ is shown by thermodynamical considerations to be

$r_{\mathrm{c}}=\frac{2 \sigma}{P_{\mathrm{s}}-p_{\mathrm{c}}}$,

where $\sigma$ is the surface tension, and $p_{v}$ and $p_{e}$ the equilibrium vapour pressure and the expanded pressure. respectively. We estimate the critical radius at our operating conditions to be $\gtrsim 30 \AA$. We have to investigate whether a unidirectional movement of the 8 -electrons due to the electric field alters the conditions for the dissipation of heat in a sphere of this size. The relaxation time for the dissipation of heat contained in such a prenucleation volume is $\tau=r_{c}^{2} / D$, where $D$ is the diffusion coefficient of heat. The latter is related to the thermal conductivity $\lambda$ by the equation $D=\lambda / \rho c_{r}$. where $\rho$ is the density of the liquid and $c_{p}$ the specific heat at constant pressure. For our operating temperature we use $\lambda=65 \times 10^{-3} \mathrm{~W} / \mathrm{m} \cdot \mathrm{K}, c_{p}=1.72 \mathrm{~J} / \mathrm{g} \cdot \mathrm{K}$ [16] and find $\tau=2.4 \times 10^{-10} \mathrm{~s}$.

The drift distance of $\delta$-electrons within this relaxation time can be estimated, assuming in our case an electric field of $2 \mathrm{kV} / \mathrm{cm}$ and a drift velocity of $v_{\mathrm{d}} \approx 2$ $\times 10^{5} \mathrm{~cm} / \mathrm{s}$, as measured at the normal boiling point and neglecting its temperature dependency [12]. We obtain $s=v_{\mathrm{d}} \cdot \tau \simeq 5 \times 10^{-5} \mathrm{~cm}$, a displacement which is considerably larger than the critical bubble diameter of $\varnothing \gtrsim 6 \times 10^{-7} \mathrm{~cm}$. The $\delta$-election would leave the prenuclear volume if it had its centre at the origin of ionization. However, as shown by Monte Carlo calculations for hydrogen and deuterium [17], the size of the excitation/ionization cascade during the slow-down of $\delta$-electrons exceeds anyway such a volume, and only at its end, when the electrons have travelled a distance of $s \simeq 3 \times 10^{-6} \mathrm{~cm}$, does the densest area of excitation remain inside a prenuclear volume. Similar behaviour may be expected for liquid argon, the difference as compared with the lighter cryogenic liquids being its higher density, its smaller critical bubble radius, and that during calculations the Auger effect should be considered in addition to excitation and ionization. Since we do not see any decrease of the bubble density in the presence of the eiectric field, we conclude that the unidirectional component of the electron's movement. caused by the electric field, only displaces the position of the prenuclear volume in space. This displacement along the electric field lines can be neglected during the reconstruction of particle tracks since it is by orders of magnitude smaller than any displacement of macroscopic bubbles due to buoyancy forces, liquid movement and turbulances.

(b) There could also be an increast of bubble density with electric field, because the amount of energy going into scintillation decreases with field strength [18] due to the escape of electrons and holes from recombina- 
tion. More energy could be available for ionization and, hence, for bubble formation. Scintillation light produced by $200 \mathrm{MeV} / \mathrm{c}$ pions has been measured in our detector [3], however, until now without electric field. Tests are being prepared to operate the charge collection device and light detection simultaneously, and to measure the field dependency of the scintillation signal.

At present we also cannot rule out the possibility that both effects may be actually there, but that they cancel each other.

\section{Conclusions}

It is shown that charge collection during bubble chamber operation of a liquid argon detector is possible, but thit noise still affects adversely the measurements, particularly during long beam spills. The dist'rbance comes mainly from the change of capacitance during the expansion, whereas most of the other sources were virtually eliminated during our tests. It is demonstrated that noise becomes smaller with longer expansion-recompression cycles, favouring the prospects for very large chambers of this type. Further improvements will be obtained using the scintillation signal as trigger of a gate for the charge collection electronics, and by a modification of the layout of the electrodes. The presence of the high electric field ( $10 \mathrm{kV}$ on the wire) does probably not alter bubble densities along particle tracks.

The authors are greatly indebted to many people in the BEBC Group at CERN, in particular to A. Carrere. P. Dupont, P. Rada and D. Voillat for their dedicated help during and in preparation of the runs of the chamber, to B. Allardyce, K. Gase and F. Schöfel and the operators of the synchrocyclotron for their invaluable assistance during the installation and runs. to $C$. Marin for mechanical constructions, and to A. Minten and $\mathbf{H}$. Wenninger for continuous support. We also like to thank W. Willis for useful discussions on calorimetry and T. Doke for criticism and suggestions.

\section{References}

[1] G. Harigel, H. Kautzky, P. McIntyre and A. van Ginneken. Fermilab Propcial 601 (1978).

[2] G. Hariget, C.Baltay and $M$. Hibbs, Columbia University. Nevis-Report. New York (1982).

[3] J.C. Berset. M. Burns, K. Geissler, G. Harigel, J. Lindswy. G. Linser and F. Schenk, submitted to Nucl. Instr. and Meth.

[4] G. Harigel, G. Linser and F. Schenk. Nucl. Instr. and Meth. 187 (1981) 363.

[5] C. Brassard, Nucl. Instr. and Meth. 162 (1979) 29.

[6] T. Doke, Portugal. Phys. Vol. 12 (1981) 9.

[7] H.H. Chen and J.F. Lathrop. Nucl. Instr. and Meth. 150 (1978) 585.

[8] H.H. Clien and P.J. Doe. IEEE Trans. on Nucl. Sci. NS·28, No. I (1981) 454.

[9] J. Lindsay, private communication (1981).

[10] C. Kourkoumelis, A Study of $J / \psi$ production in pp collisions at the CERN ISR using liquid argon calorimeters and lithium/xenon transition radiation detectors, Yellow Report CERN 77-06 (1977).

[11] H. Schnyders, S.A. Rice and L. Meyer. Phys. Rev. Al4 (1976) 438.

[12] K. Yoshino, U. Sowada and W.F. Schmidt. Phys. Rev. Al4 (1976) 438 .

[13] W. Hoimann, U. Klein, M. Schulz, J. Spengler and D. Wegener, Nucl. Instr. and Meth. 135 (1976) IS1.

[14] J. Hofmann and E. Hugentobler. Helvetica Physica Actis 38 (1965) 783.

[15] T. Doke. private comntunication (1982).

[16] B.J. Bailey and K. Kellner, Physica 39 (1988) 444: N.B. Vargaftik, Tables on the thermophysical properties of liquids and gases (John Wiley. New York. London. Sidney. Toronto. 1975) second ed., (Hemisph. Publ Corp. Advances in thermal engineering).

[17] A.G. Tenner. Structure of tracks of charged particks in condensed matter, in Proc. Gray Memorial Confereace (Bubble chambers), Cambridge. April (1969) p. 44

[18] S. Kubota. A. Nakamoto. T. Takahashi. T. Hamada E. Shibamura, M. Miyahima. K. Masuda and T. Doke. Phass Rev. B17 (1978) 2762. 